\title{
Multidimensional ultrametric pseudodifferential equations
}

\author{
S.Albeverio, S.V.Kozyrev
}

July 6, 2021

\begin{abstract}
We develop an analysis of wavelets and pseudodifferential operators on multidimensional ultrametric spaces which are defined as products of locally compact ultrametric spaces. We introduce bases of wavelets, spaces of generalized functions and Lizorkin generalized functions on multidimensional ultrametric spaces.

We also consider some family of pseudodifferential operators on multidimensional ultrametric spaces. The notions of Cauchy problem for ultrametric pseudodifferential equations and of ultrametric characteristics are introduced. A theorem about existence and uniqueness of the solution for the Cauchy problem (the analogue of the Kovalevskaya theorem) is proven.
\end{abstract}

Keywords: Wavelets, pseudodifferential operators, multidimensional ultrametric spaces, Lizorkin generalized functions, Cauchy problems, Kovalevskaya theorem.

AMS-classification: 26E30, 26A33, 45N05, 45E99, 42C40, 46F12

\section{Introduction}

Ultrametric and $p$-adic analysis in interaction with mathematical physics constitutes a broad field of research, see e.g. [1], 2], 3], 4], [5], 6], [7. We mention the $p$-adic wavelets introduced in [8] and generalized in [9] to some family of abelian groups. The generalization of $p$-adic wavelets to the case of underlying general locally compact ultrametric spaces [10], [11], [12] is a crucial tool for the approach of the present paper. In [13] new examples of $p$-adic wavelets were constructed and it was shown that the basis of $p$-adic Haar wavelets is not unique. In [14] it was shown that the continuous $p$-adic wavelet transform coincides with the expansion in the series on $p$-adic wavelets from the basis constructed in 8 .

An important notion of ultrametric analysis is one of the ultrametric pseudodifferential operators, defined by the following formula, see [10], 11], [12, [15, (and also the Appendix 2 , for details)

$$
T f(x)=\int_{X} T(\sup (x-y))(f(x)-f(y)) d \nu(y) .
$$

The above operator acts in the space $L^{2}(X, \nu)$ of complex valued functions which are quadratically integrable with respect to the measure $\nu$ on a locally compact ultrametric space $X$. It 
was found [10], [11], [12] that operators of this form are diagonal with respect to a natural basis consisting of ultrametric wavelets in $L^{2}(X, \nu)$, and one can compute the spectra of these operators using an explicit formula. Moreover, the eigenvalues of the above operator are in one-to-one correspondence with the balls of non-zero diameters in the space $X$.

In the present paper we investigate several topics of ultrametric analysis.

First, we consider multidimensional ultrametric spaces, which are defined as products $X=X^{1} \times \ldots \times X^{n}$ of locally compact ultrametric spaces with the product measure $\nu=$ $\nu^{1} \times \ldots \times \nu^{n}$. We introduce bases of wavelets, spaces of generalized functions and Lizorkin's generalized functions on multidimensional ultrametric spaces.

The space of Lizorkin generalized functions in the $p$-adic case was introduced in [16]. We generalize this definition to the general ultrametric case. In particular, we find the characterization of Lizorkin generalized functions by formal series of ultrametric wavelets.

Second, we consider the family of pseudodifferential operators on multidimensional ultrametric spaces, which contains polynomials in one-dimensional ultrametric pseudodifferential operators. These operators are diagonal in the mentioned above bases of multidimensional ultrametric wavelets, and moreover, the eigenvalues are in one-to-one correspondence with the products of balls $\mathbf{I}=I^{1} \times \ldots \times I^{n}$, where $I^{j}$ is a ball in $X^{j}$. We introduce the notion of ultrametric characteristics — we say that the product of balls $\mathbf{I}$ is characteristic for the operator $T$ if the corresponding eigenvalue $\lambda_{\mathbf{I}}$ of the operator $T$ is equal to zero.

Third, we introduce the following notion of Cauchy problem for ultrametric pseudodifferential equations: we say that $u$ is a solution of Cauchy problem

$$
T u=f
$$

if $T$ is an ultrametric pseudodifferential operator, $f$ lies in Lizorkin space of generalized functions, $u$ lies in the space of generalized functions and satisfies the initial condition $u\left(\chi_{\mathbf{I}}\right)=$ $u_{0} \nu(\mathbf{I})$, where $\chi_{\mathbf{I}}$ is the characteristic function of $\mathbf{I}$ and $u_{0}$ is a complex number.

We prove theorem about existence of the solution for the Cauchy problem (the analogue of the Kovalevskaya theorem). We show that the Cauchy problem possesses a unique solution if there are no characteristics.

The main technical tool for the investigations of the present paper is the expansion of generalized function on an ultrametric space to a series of ultrametric wavelets, given by Lemma 3 in the one dimensional case and by Lemma 8 in the multidimensional case.

The structure of the present paper is as follows.

In section 2 we discuss Lizorkin space of generalized functions on an ultrametric space and show that these generalized functions can be considered as formal series over ultrametric wavelets.

In section 3 we find expansion of a generalized function on a regular ultrametric space in a series over wavelets, introduce the notion of Cauchy problem for pseudodifferential equations on an ultrametric space, and prove the theorem about the existence and uniqueness of a solution for the Cauchy problem.

In section 4 we build bases of wavelets on multidimensional ultrametric spaces and discuss the corresponding hypergraphs.

In section 5 we discuss generalized functions on multidimensional ultrametric spaces.

In section 6 we find expansion of generalized functions in series of ultrametric wavelets in the multidimensional case. 
In section 7 we introduce a family of multidimensional ultrametric pseudodifferential operators and introduce the notion of an ultrametric characteristic.

In section 8 we prove the theorem about existence and uniqueness of the solution for Cauchy problem for multidimensional ultrametric pseudodifferential equations (the analogue of the Kovalevskaya theorem).

In section 9 (Appendix 1) we recall the notion of a characteristic for (real) equations in partial derivatives.

In section 10 (Appendix 2) we recall some results on analysis of wavelets and pseudodifferential operators on ultrametric spaces.

\section{Lizorkin space of generalized functions}

Consider the space $D_{0}(X) \subset D(X)$ of mean zero test functions on a regular ultrametric space $X$. This space possesses the filtration by subspaces $D_{0}(\mathcal{S})$ of mean zero test functions in $D(\mathcal{S})$. It is easy to see that the space $D_{0}(\mathcal{S})$ is the linear span of wavelets $\Psi_{I j}$ where $I \in \mathcal{S}$ runs over all non-minimal vertices in $\mathcal{S}$. The space $D_{0}(X)$ is spanned by all ultrametric wavelets. The topology on $D_{0}(X)$ is introduced in the obvious way.

Definition 1 Lizorkin space $D_{0}^{\prime}(X)$ of generalized functions is the space of linear functionals on the space $D_{0}(X)$ of mean zero test functions.

We have the following two characterizations of Lizorkin space.

1) Since the space $D_{0}(X)$ is exactly the space of test functions which lie in the kernel of the constants (we consider the constants as generalized functions), the space $D_{0}^{\prime}(X)$ coincides with the space of equivalence classes of generalized functions which are equal up to addition of a constant. In particular, any generalized function in $D^{\prime}(X)$ can be considered also as a generalized function in Lizorkin space $D_{0}^{\prime}(X)$.

2) Since the space $D_{0}(X)$ is a space of finite linear combinations of ultrametric wavelets from the orthonormal basis $\left\{\Psi_{I j}\right\}$ in $L^{2}(X, \nu)$, the space $D_{0}^{\prime}(X)$ of linear functionals on $D_{0}(X)$ can be identified with the space of formal series over ultrametric wavelets, with the following action on $D_{0}(X)$.

Let $f \in D_{0}(X)$ have the form of a finite linear combination of wavelets

$$
f=\sum_{I j} f_{I j} \Psi_{I j}, \quad f_{I j}=\left\langle\Psi_{I j}, f\right\rangle
$$

where $\langle\cdot, \cdot\rangle$ is the scalar product in $L^{2}(X, \nu)$ :

$$
\langle f, g\rangle=\int_{X} \overline{f(x)} g(x) d \nu(x)
$$

where $\bar{f}$ denotes the complex conjugate to $f$.

Let $\phi \in D_{0}^{\prime}(X)$ have the form of a series over wavelets:

$$
\phi=\sum_{I^{\prime} j^{\prime}} \phi_{I^{\prime} j^{\prime}} \Psi_{I^{\prime} j^{\prime}}, \quad \phi_{I^{\prime} j^{\prime}}=\phi\left(\overline{\Psi_{I^{\prime} j^{\prime}}}\right) .
$$

Then the action of $\phi$ on $f$ is given by the following finite linear combination

$$
\phi(f)=\sum_{I j} \phi_{I j} f_{I j} .
$$




\section{Cauchy problem in the one dimensional case}

In the previous section we discussed expansion of generalized function from Lizorkin space $D_{0}^{\prime}(X)$ in series over ultrametric wavelets. In the present section we build the analogous expansion for generalized functions in $D^{\prime}(X)$.

Lemma 2 Assume that for a ball $I_{0} \subset X$ its $\nu$-measure is positive: $\nu\left(I_{0}\right)>0$.

Then there exists the unique generalized function $u \in D^{\prime}(X)$ such that

$$
u\left(\chi_{I_{0}}\right)=u_{0} \nu\left(I_{0}\right), \quad u\left(\overline{\Psi_{I j}}\right)=u_{I j}, \quad \forall I, j .
$$

Proof We note that for any regular subtree $\mathcal{S} \subset \mathcal{T}(X)$ (see the Appendix 2 for the notations) the corresponding space $D(\mathcal{S})$ of test functions is an orthogonal sum of the space $D_{0}(\mathcal{S})$ of mean zero test functions (spanned by the corresponding wavelets) and the one dimensional linear space spanned by the characteristic function $\chi_{\sup (\mathcal{S})}$ of the ball $\sup (\mathcal{S})$. The ball $\sup (\mathcal{S})$ is the largest ball in the set $\mathcal{S}$ and is the support of $D(\mathcal{S})$.

If a ball $I_{0} \in \mathcal{S}$ has positive measure $\nu\left(I_{0}\right)>0$, then its characteristic function $\chi_{I_{0}}$ is not orthogonal to the characteristic function $\chi_{\sup (\mathcal{S})}$. Therefore the union of the basis of wavelets in $D_{0}(\mathcal{S})$ and the characteristic function $\chi_{I_{0}}$ is a complete set in $D(\mathcal{S})$.

Therefore the action of an arbitrary generalized function $u$ in $D(\mathcal{S})$ can be defined by its application to all wavelets and to a characteristic function $\chi_{I_{0}}, \nu\left(I_{0}\right)>0$. This finishes the proof of the lemma.

Let us prove the following important lemma about the expansion of generalized functions in $D^{\prime}(X)$ into series over wavelets.

Lemma 3 Assume that for a ball $I_{0} \subset X$ its $\nu$-measure is positive: $\nu\left(I_{0}\right)>0$.

Then the series

$$
u=u_{0}+\sum_{I j} u_{I j}\left(\Psi_{I j}-\frac{1}{\nu\left(I_{0}\right)} \Psi_{I j}\left(\chi_{I_{0}}\right)\right)
$$

is the generalized function $u \in D^{\prime}(X)$ which satisfies the condition

$$
u\left(\chi_{I_{0}}\right)=u_{0} \nu\left(I_{0}\right), \quad u\left(\overline{\Psi_{I j}}\right)=u_{I j}
$$

Moreover an arbitrary $u \in D^{\prime}(X)$ satisfying (2) has the form (1).

Proof To check that the series (1) is indeed a generalized function in $D^{\prime}(X)$, we have to prove that application of this series to any characteristic function $\chi_{J_{0}}$ gives a convergent series. We have

$$
u\left(\chi_{J_{0}}\right)=u_{0} \nu\left(J_{0}\right)+\sum_{I j} u_{I j}\left(\Psi_{I j}\left(\chi_{J_{0}}\right)-\frac{\nu\left(J_{0}\right)}{\nu\left(I_{0}\right)} \Psi_{I j}\left(\chi_{I_{0}}\right)\right)
$$

We see that for sufficiently large $I$, namely for $I>\sup \left(J_{0}, I_{0}\right)$, we get

$$
\nu^{-1}\left(I_{0}\right) \Psi_{I j}\left(\chi_{I_{0}}\right)=\Psi_{I j}(x)
$$


where $x \in I_{0}$. Analogously

$$
\Psi_{I j}\left(\chi_{J_{0}}\right)=\nu\left(J_{0}\right) \Psi_{I j}\left(x^{\prime}\right)
$$

where $x^{\prime} \in J_{0}$.

Since for $I>\sup \left(J_{0}, I_{0}\right)$ we have $\Psi_{I j}(x)=\Psi_{I j}\left(x^{\prime}\right)$, the corresponding terms in the series above cancel, and the series will take the form of the finite sum

$$
u\left(\chi_{J_{0}}\right)=u_{0} \nu\left(J_{0}\right)+\sum_{I j: J_{0}<I \leq \sup \left(J_{0}, I_{0}\right)} u_{I j} \Psi_{I j}\left(\chi_{J_{0}}\right)-\frac{\nu\left(J_{0}\right)}{\nu\left(I_{0}\right)} \sum_{I j: I_{0}<I \leq \sup \left(J_{0}, I_{0}\right)} u_{I j} \Psi_{I j}\left(\chi_{I_{0}}\right) .
$$

This proves that the series (1) is a generalized function in $D^{\prime}(X)$.

The application of (11) on $\chi_{I_{0}}$ proves that $u$ satisfies the condition $u\left(\chi_{I_{0}}\right)=u_{0} \nu\left(I_{0}\right)$.

Analogously, the application of (11) to $\overline{\Psi_{I j}}$, taking into account that

$$
\Psi_{I^{\prime} j^{\prime}}\left(\overline{\Psi_{I j}}\right)=\left\langle\Psi_{I j}, \Psi_{I^{\prime} j^{\prime}}\right\rangle=\delta_{I I^{\prime}} \delta_{j j^{\prime}}
$$

proves that $u$ satisfies the condition $u_{I j}=u\left(\overline{\Psi_{I j}}\right)$.

Then by applying Lemma 2 we prove that an arbitrary $u \in D^{\prime}(X)$ satisfying (2) has the form (11). This finishes the proof of the lemma.

Remark We understand the series (11) as a limit with respect to a filtration, i.e. a finite sum of the series depends on the regular subtree $\mathcal{S}$, and the series is a limit of finite sums with $\mathcal{S} \rightarrow \mathcal{T}(X)$.

Let us consider the ultrametric pseudodifferential operator $T$ on the regular ultrametric space $X$ with the eigenvalues $\lambda_{I}$ in the wavelet basis.

For an arbitrary generalized function $u \in D^{\prime}(X)$ the application of a pseudodifferential operator $T$ to this function gives a generalized function in Lizorkin space $T u \in D_{0}^{\prime}(X)$, with the action on wavelets defined as follows

$$
T u\left(\overline{\Psi_{I j}}\right)=u\left(\overline{T^{*} \Psi_{I j}}\right)=\lambda_{I} u\left(\overline{\Psi_{I j}}\right) .
$$

This formula is a direct analogue of the well known formula of real analysis which defines the derivative of a generalized function by $u^{\prime}(\phi)=-u\left(\phi^{\prime}\right)$, where $u$ is a generalized function and $\phi$ is a test function on the real line.

Therefore ultrametric pseudodifferential operators can be considered as linear maps $D^{\prime}(X) \rightarrow D_{0}^{\prime}(X)$, or, in the analogous way, as linear maps $D_{0}^{\prime}(X) \rightarrow D_{0}^{\prime}(X)$. Note that in general it is not possible to consider a pseudodifferential operator as a map $D^{\prime}(X) \rightarrow D^{\prime}(X)$.

Definition 4 We say that a generalized function $u \in D^{\prime}(X)$ is a solution of Cauchy problem if

$$
T u=f,
$$

where $f \in D_{0}^{\prime}(X)$ lies in Lizorkin space, and $u$ satisfies the initial condition: $u\left(\chi_{I_{0}}\right)=$ $u_{0} \nu\left(I_{0}\right)$, for the characteristic function $\chi_{I_{0}}$ of some ball $I_{0} \subset X, \nu\left(I_{0}\right)>0$.

It is easy to see that there exist necessary conditions for solvability of (3): if $\lambda_{I}=0$ for some $I$ then all $f_{I j}=f\left(\overline{\Psi_{I j}}\right)=0$. 
Theorem 5 Let the Lizorkin generalized function $f$ at the RHS of (3) satisfy the necessary conditions $f_{I j}=f\left(\overline{\Psi_{I j}}\right)=0$ for $I$ for which $\lambda_{I}=0$ and for all corresponding indices $j$.

Then there exists a solution of the Cauchy problem (3), and an arbitrary solution $u$ of the Cauchy problem (3) will be given by the formula

$$
u=u_{0}+\sum_{I j} \frac{1}{\lambda_{I}} f_{I j}\left(\Psi_{I j}-\frac{1}{\nu\left(I_{0}\right)} \Psi_{I j}\left(\chi_{I_{0}}\right)\right)+\sum_{J j} u_{J j}\left(\Psi_{J j}-\frac{1}{\nu\left(I_{0}\right)} \Psi_{J j}\left(\chi_{I_{0}}\right)\right) .
$$

The summation runs over $I$ for which $\lambda_{I} \neq 0$ and over $J$ for which $\lambda_{J}=0$. Here $f_{I j}=f\left(\overline{\Psi_{I j}}\right)$ and $u_{J j}$ are arbitrary.

Proof By Lemma 3 a generalized function $u \in D^{\prime}(X)$ satisfying the initial condition $u\left(\chi_{I_{0}}\right)=u_{0} \nu\left(I_{0}\right)$ has the form (11).

Since the operator $T$ is diagonal in the basis of wavelets, applying $T$ to (1) we get from (3) for $I$, for which $\lambda_{I} \neq 0$

$$
u_{I j}=\frac{1}{\lambda_{I}} f_{I j}
$$

Application of the operator $T$ to (44) proves that $u$ is a solution of (3)). This finishes the proof of the theorem.

\section{Product of ultrametric spaces and wavelets}

Assume we have several regular ultrametric spaces $X^{1}, \ldots, X^{n}$ with Borel measures $\nu^{1}, \ldots, \nu^{n}$. Let us consider the product space $X=X^{1} \times \ldots \times X^{n}$ with the product measure $\nu=$ $\nu^{1} \times \ldots \times \nu^{n}$.

Each of the spaces $X^{i}$ is dual to the corresponding directed tree $\mathcal{T}\left(X^{i}\right)$. The tree $\mathcal{T}\left(X^{i}\right)$ is defined by the set of vertices (balls), and a family of two-element subsets (edges). The tree $\mathcal{T}\left(X^{i}\right)$ is used in the construction of the corresponding basis of ultrametric wavelets.

In the present section we construct an object, called a hypergraph, which we will use in the procedure of construction of the basis of multidimensional wavelets on the space $X=X^{1} \times \ldots \times X^{n}$. In general, a hypergraph is a set with the elements called vertices, and several families of subsets: family of pairs, family of $k$-tuples, etc. The family of subsets containing $k$ elements called the family of edges of the order $k-1$. In particular, a graph is a hypergraph with the family of pairs of vertices, called the family of edges (of the order one).

We define the hypergraph $\mathcal{T}=\mathcal{T}\left(X^{1}\right) \times \ldots \times \mathcal{T}\left(X^{n}\right)$ as follows. We have the set of vertices, which is the set of $n$-tuples $\mathbf{I}=\left(I^{1}, \ldots, I^{n}\right)$, where $I^{j}$ is a vertex in $\mathcal{T}\left(X^{j}\right)$. We will also use the notation $\mathbf{I}=I^{1} \times \ldots \times I^{n}$. We denote by $\chi_{\mathbf{I}}$ the function on $X=X^{1} \times \ldots \times X^{n}$ which is equal to the characteristic function of the product of balls $\mathbf{I}$.

Then, we have the set of one-dimensional edges - the set of pairs $(\mathbf{I}, \mathbf{J})$, where

$$
\mathbf{I}=\left(I^{1}, \ldots, I^{k}, \ldots I^{n}\right), \quad \mathbf{J}=\left(I^{1}, \ldots, J^{k}, \ldots I^{n}\right)
$$

for some $k$ (i.e. one element in the tuples differs), and, moreover $I^{k}$ and $J^{k}$ belong to some edge in $\mathcal{T}\left(X^{k}\right)$. One can say that the edge $(\mathbf{I}, \mathbf{J})$ can be identified with the direct product of 
the edge $\left(I^{k}, J^{k}\right)$ in $\mathcal{T}\left(X^{k}\right)$ and the product of vertices $I^{1} \times \ldots I^{k-1} \times I^{k+1} \ldots I^{n}$ in $\mathcal{T}\left(X^{j}\right)$, $j \neq k$. Since all edges $\mathcal{T}\left(X^{k}\right)$ are ordered sets, the same will hold for the edge $(\mathbf{I}, \mathbf{J})$ in $\mathcal{T}$. This defines our partial order in the hypergraph $\mathcal{T}$.

The set of two-dimensional edges contains quadruples $(\mathbf{A}, \mathbf{B}, \mathbf{C}, \mathbf{D})$, where $(\mathbf{A}, \mathbf{B}),(\mathbf{A}, \mathbf{C})$, $(\mathbf{B}, \mathbf{D})$ and $(\mathbf{C}, \mathbf{D})$ are one-dimensional edges. Thus a two-dimensional edge is a square where the vertices are vertices in $\mathcal{T}$ and the sides are one-dimensional edges in $\mathcal{T}$. In more details this construction is as follows: let

$$
\begin{aligned}
& \mathbf{A}=\left(A^{1}, \ldots, A^{i}, \ldots, A^{j}, \ldots, A^{n}\right), \\
& \mathbf{B}=\left(A^{1}, \ldots, B^{i}, \ldots, A^{j}, \ldots, A^{n}\right),
\end{aligned}
$$

(since $(\mathbf{A}, \mathbf{B})$ is a one-dimensional edge) and

$$
\mathbf{C}=\left(A^{1}, \ldots, A^{i}, \ldots, C^{j}, \ldots, A^{n}\right),
$$

then

$$
\mathbf{D}=\left(A^{1}, \ldots, B^{i}, \ldots, C^{j}, \ldots, A^{n}\right) .
$$

Analogously, a $d$-dimensional edge in $\mathcal{T}, d=0,1, \ldots, n$ is a $d$-dimensional cube with the sides being $d$-1-dimensional edges in $\mathcal{T}$. It is easy to see that a $d$-dimensional edge in $\mathcal{T}$ has the order $2^{d}-1$ (i.e. contains $2^{d}$ vertices).

By construction, since a $d$-dimensional edge is a product of the one dimensional edges which are ordered sets, it possesses a natural partial order. A $d$-dimensional edge contains the largest and the smallest vertices. These vertices are the opposite vertices in the cube (i.e. the line connecting these vertices is the main diagonal in the cube). The different edges may intersect and the partial orders in the intersection in both edges coincide.

We introduce the following notion: we say that the vertex $\mathbf{I}=\left(I^{1}, \ldots, I^{n}\right)$ is sufficiently larger than the vertex $\mathbf{J}=\left(J^{1}, \ldots, J^{n}\right)$ and we write:

$$
\text { I }>>\text { J }
$$

if $\forall i=1, \ldots, n$ we have

$$
I^{i}>J^{i} .
$$

For any two vertices $\mathbf{I}=\left(I^{1}, \ldots, I^{n}\right)$ and $\mathbf{J}=\left(J^{1}, \ldots, J^{n}\right)$ there exists the unique supremum, i.e. the minimal vertex that is larger than both $\mathbf{I}$ and $\mathbf{J}$ :

$$
\sup (\mathbf{I}, \mathbf{J})=\left(\sup \left(I^{1}, J^{1}\right), \ldots, \sup \left(I^{n}, J^{n}\right)\right)
$$

Therefore $\mathcal{T}$ is a directed set.

For any two vertices in $\mathcal{T}$ there exists a path (i.e. a sequence of one-dimensional edges) in $\mathcal{T}$, connecting the edges. This path in general is not unique.

Let us remind that the branching index $p_{I}$ of a vertex $I$ in the tree of balls $\mathcal{T}(X)$, corresponding to a regular ultrametric space $X$, is equal to the number of maximal subballs in the ball $I$ in $X$.

Analogously, we will say that the branching index of the vertex $\mathbf{I}=\left(I^{1}, \ldots, I^{k}, \ldots I^{n}\right)$ in $\mathcal{T}$ is equal to $\left(p_{I^{1}}, \ldots, p_{I^{n}}\right)$, where $p_{I^{j}}$ is the branching index of $I^{j}$. 
The $k$-dimensional edge with the beginning in $\mathbf{I}$ is called decreasing if $\mathbf{I}$ is the maximal vertex in the edge. The number of decreasing edges of maximal dimension with the beginning in the vertex $\mathbf{I}=\left(I^{1}, \ldots, I^{k}, \ldots I^{n}\right)$ is equal to the product of branching indices

$$
\prod_{j} p_{I^{j}}
$$

the product being taken over all non-minimal $I^{j}$ in $\mathbf{I}$ (with non-zero branching indices). The dimension of decreasing edges with maximal dimension which begin in $\mathbf{I}$ is equal to the number of non-minimal $I^{j}$ in $\mathbf{I}$.

If all $I^{j}$ in $\mathbf{I}$ are non-minimal, we will say that the vertex $I$ is generic. In this case decreasing edges of maximal dimension with the beginning in $\mathbf{I}$ have the dimension $n$.

Let us discuss the wavelet space corresponding to a vertex $\mathbf{I}$ in the hypergraph $\mathcal{T}$. If $\mathbf{I}=\left(I^{1}, \ldots, I^{k}, \ldots I^{n}\right)$ is a generic vertex, then we define the wavelet space $V^{0}(\mathbf{I})$ as the tensor product of wavelet spaces corresponding to vertices $I^{j}$ in $\mathcal{T}\left(X^{j}\right)$ :

$$
V^{0}(\mathbf{I})=\otimes_{j} V^{0}\left(I^{j}\right)
$$

The space $V^{0}(\mathbf{I})$ possesses the orthonormal basis consisting of tensor products of wavelets

$$
\Psi_{\mathbf{I j}}=\otimes_{k} \Psi_{I^{k} j^{k}}
$$

where $\left\{\Psi_{I^{k} j^{k}}\right\}$ is the orthonormal basis in the wavelet space corresponding to the vertex $I^{k} \in \mathcal{T}\left(X^{k}\right), \mathbf{I}=\left(I^{1}, \ldots, I^{n}\right), \mathbf{j}=\left(j^{1}, \ldots, j^{n}\right)$.

We will use the hypergraph $\mathcal{T}(X)$ to construct the wavelet bases in $L^{2}(X, \nu)$. We will get the following problem. It might happen that some of the spaces $X^{i}$ could have a finite volume $\nu^{i}\left(X^{i}\right)<\infty$. In this case, in order to obtain basis in $L^{2}\left(X^{i}, \nu^{i}\right)$, we should add a constant function to the set of wavelets, corresponding to non-minimal balls $I^{i} \in \mathcal{T}\left(X^{i}\right)$. The wavelets $\Psi_{I j}$ correspond to the ball $I \in \mathcal{T}\left(X^{i}\right)$, but the constant function in general can not be put into correspondence to any of the balls in $\mathcal{T}\left(X^{i}\right)$.

Let us perform the following construction. If the volume $\nu^{i}\left(X^{i}\right)=A>0$ is finite, let us add to the tree $\mathcal{T}\left(X^{i}\right)$ of non-minimal balls the vertex $K^{(i)}$, which is larger than all vertices in $\mathcal{T}\left(X^{i}\right)$. We denote by $\widetilde{\mathcal{T}}\left(X^{i}\right)=\mathcal{T}\left(X^{i}\right) \cup K^{(i)}$, this is a directed set (but as a graph this set is not connected, because we do not assume existence of edges containing vertex $K^{(i)}$ ). Then the basis of ultrametric wavelets in $L^{2}\left(X^{i}, \nu^{i}\right)$ could be described as a union of wavelets corresponding to vertices in $\mathcal{T}\left(X^{i}\right)$, and a constant function $\Psi_{K^{(i)}}=A^{-1 / 2}$ in $L^{2}\left(X^{i}, \nu^{i}\right)$ which corresponds to vertex $K^{(i)}$ (we consider this vertex as generic). If the measure of $X^{i}$ is infinite, we define $\tilde{\mathcal{T}}\left(X^{i}\right)=\mathcal{T}\left(X^{i}\right)$.

We will call the augmented hypergraph $\widetilde{\mathcal{T}}$ the hypergraph

$$
\tilde{\mathcal{T}}=\tilde{\mathcal{T}}\left(X^{1}\right) \times \ldots \times \tilde{\mathcal{T}}\left(X^{n}\right) .
$$

The edges and partial order in the augmented hypergraph are introduced in the same way as in the hypergraph $\mathcal{T}$. Let us note that the augmented hypergraph $\widetilde{\mathcal{T}}$, in general, is not connected.

We consider multidimensional wavelets defined by the formula (15), whose vertices I belong to the augmented hypergraph $\widetilde{\mathcal{T}}$. Let us formulate the following theorem about the multidimensional basis of ultrametric wavelets. 
Theorem 6 1) Let the volumes $\nu^{i}\left(X^{i}\right), i=1, \ldots, n$ be infinite. Then the union $\left\{\Psi_{\mathbf{I j}}\right\}$ of the bases in $V^{0}(\mathbf{I})$ over all generic $\mathbf{I} \in \mathcal{T}$ is an orthonormal basis in $L^{2}(X, \nu)$.

2) Assume that some of the volumes $\nu^{i}\left(X^{i}\right)=A_{i}$ are finite. Then the set $\left\{\Psi_{\mathbf{I} \mathbf{j}}\right\}$, where I runs over generic vertices in the augmented hypergraph $\tilde{\mathcal{T}}$ is an orthonormal basis in $L^{2}(X, \nu)$.

\section{Multidimensional generalized functions}

For the multidimensional ultrametric space $X=X^{1} \times \ldots \times X^{n}$ we define the space of test functions $D(X)$ as the tensor product of one-dimensional spaces of test functions:

$$
D(X)=\otimes_{i=1}^{n} D\left(X^{i}\right)
$$

Analogously, we define Lizorkin space of test functions $D_{0}(X)$ as

$$
D_{0}(X)=\otimes_{i=1}^{n} D_{0}\left(X^{i}\right)
$$

Equivalently, the space $D_{0}(X)$ can be defined as the space of test functions satisfying

$$
\int_{X^{i}} f\left(x^{1}, \ldots, x^{i}, \ldots, x^{n}\right) d \nu^{i}\left(x^{i}\right)=0, \quad \forall i=1, \ldots, n .
$$

Topologies in the spaces $D(X)$ and $D_{0}(X)$ are defined in the standard way. Since $D\left(X^{i}\right)$ are filtrated by $D\left(\mathcal{S}^{i}\right)$, the space $D(X)$ will be filtrated by finite dimensional subspaces

$$
D\left(\mathcal{S}^{1} \times \ldots \times \mathcal{S}^{n}\right)=D\left(\mathcal{S}^{1}\right) \otimes \ldots \otimes D\left(\mathcal{S}^{n}\right) .
$$

A sequence in $D(X)$ converges if it belongs to some subspace $D(\mathcal{S})$ and converges as a sequence in this finite dimensional space. The analogous statements will hold for $D_{0}(X)$.

The space $D^{\prime}(X)$ of generalized functions is the space of linear functionals on $D(X)$. The space $D^{\prime}(X)$ possesses the topology of weak convergence of the functionals.

Lizorkin space of generalized functions $D_{0}^{\prime}(X)$ is the space of linear functionals on $D_{0}(X)$ with the topology of weak convergence.

Since the space $D_{0}(X)$ can be identified with the space of finite linear combinations of multidimensional wavelets $\Psi_{\mathbf{I} \mathbf{j}}, \mathbf{I} \in \mathcal{T}$, Lizorkin space $D_{0}^{\prime}(X)$ can be considered as the space of series over wavelets

$$
f=\sum_{\mathbf{I} \mathbf{j}, \mathbf{I} \in \mathcal{T}} f_{\mathbf{I} \mathbf{j}} \Psi_{\mathbf{I} \mathbf{j}}, \quad f_{\mathbf{I} \mathbf{j}}=f\left(\overline{\Psi_{\mathbf{I j}}}\right) .
$$

Here $\mathbf{I}$ runs over generic vertices in the hypergraph $\mathcal{T}$.

Analogously, Lizorkin space of generalized functions can be identified with the factorspace $D^{\prime}(X) / D_{0}^{\perp}(X)$, where $D_{0}^{\perp}(X)$ is the subspace of generalized functions which kill all test functions in $D_{0}(X)$. 


\section{Expansion of generalized functions over wavelets}

Lemma 7 Assume that for a vertex $\mathbf{I}_{0} \in \mathcal{T}, \mathbf{I}_{0}=I_{0}^{1} \times \ldots \times I_{0}^{n}$ its measure is positive: $\nu\left(\mathbf{I}_{0}\right)=\prod_{i=1}^{n} \nu^{i}\left(I_{0}^{i}\right)>0$.

Denote by $\Psi_{I^{i} j^{i}}, I^{i} \in \mathcal{T}\left(X^{i}\right), j^{i}=0,1, \ldots, p_{I^{i}}-1$ the function on the ultrametric space $X^{i}$, which is defined as follows:

1) it is equal to the corresponding ultrametric wavelet for $j^{i}=1, \ldots, p_{I^{i}}-1$ and nonminimal $I^{i} \in \mathcal{T}\left(X^{i}\right)$;

2) it is equal to zero for $j^{i}=1, \ldots, p_{I^{i}}-1$ and minimal $I^{i} \in \mathcal{T}\left(X^{i}\right)$;

3) it is equal to $\chi_{I_{0}^{i}}$ for $j^{i}=0$ and $I^{i}=I_{0}^{i}$;

4) it is equal to zero for $j^{i}=0$ and $\left.I^{i} \neq I_{0}^{i}\right)$.

Define for $\mathbf{I} \in \mathcal{T}$ and $\mathbf{j}=\left(j^{1}, \ldots, j^{n}\right), j^{i}=0,1, \ldots, p_{I^{i}}-1$

$$
\Psi_{\mathbf{I j}}=\otimes_{i=1}^{n} \Psi_{I^{i} j^{i}}
$$

Then:

1) the set $\left\{\Psi_{\mathbf{I} \mathbf{j}}\right\}$ is a linearly independent and is a complete set in $D(X)$;

2) there exists a unique generalized function $u \in D^{\prime}(X)$ taking the values

$$
u\left(\overline{\Psi_{\mathbf{I j}}}\right)=u_{\mathbf{I j}} \prod_{i: j^{i}=0} \nu^{i}\left(I_{0}^{i}\right)
$$

for all $\mathbf{I}, \mathbf{j}$ (taking into account that $u_{\mathbf{I} \mathbf{j}}=0$ if the corresponding $\Psi_{\mathbf{I} \mathbf{j}}=0$ ).

Proof Let us note that the set of functions $\left\{\Psi_{\mathbf{I} \mathbf{j}}\right\}$ is the set of all pairwise tensor products of functions from the corresponding sets $\left\{\Psi_{I^{i} j_{i}}\right\}$.

In Lemma 2 it was proven that $\left\{\Psi_{I^{i} j_{i}}\right\}$ is a linearly independent complete set in $D\left(X^{i}\right)$.

Since $D(X)=\otimes_{i=1}^{n} D\left(X^{i}\right)$, the same will hold for the set $\left\{\Psi_{\mathbf{I j}}\right\}$.

Therefore any generalized function is determined by its action on $\left\{\Psi_{\mathbf{I j}}\right\}$. This finishes the proof of the lemma.

If all $j^{i}, i=1, \ldots, n$ are non-zero, the corresponding function $\Psi_{\mathbf{I j}}$ is an ultrametric wavelet. If some $j^{i}$ are zero, then $\Psi_{\mathbf{I j}}$ does not belong to the basis of wavelets.

Lemma 8 Assume that for a vertex $\mathbf{I}_{0} \in \mathcal{T}, \mathbf{I}_{0}=I_{0}^{1} \times \ldots \times I_{0}^{n}$ its measure is positive: $\nu\left(\mathbf{I}_{0}\right)=\prod_{i=1}^{n} \nu^{i}\left(I_{0}^{i}\right)>0$.

Then the series

$$
u=\sum_{\mathbf{I} \mathbf{j}} u_{\mathbf{I j}} \otimes_{i: j^{i} \neq 0}\left(\Psi_{I^{i} j^{i}}-\frac{1}{\nu^{i}\left(I_{0}^{i}\right)} \Psi_{\mathbf{I j}}\left(\chi_{I_{0}^{i}}\right)\right)
$$

is the generalized function $u \in D^{\prime}(X)$ which satisfies the condition

$$
u\left(\overline{\Psi_{\mathbf{I j}}}\right)=u_{\mathbf{I j}} \prod_{i: j^{i}=0} \nu^{i}\left(I_{0}^{i}\right)
$$

for all $\mathbf{I}, \mathbf{j}$ (again, taking into account that $u_{\mathbf{I} \mathbf{j}}=0$ if the corresponding $\Psi_{\mathbf{I j}}=0$ ).

Moreover an arbitrary $u \in D^{\prime}(X)$ satisfying (7) has the form (6). 
Proof To check that the series (6) is indeed a generalized function in $D^{\prime}(X)$, we have to prove that application of this series to any characteristic function $\chi_{\mathbf{J}_{0}}$ gives a convergent series. We have

$$
u\left(\chi_{\mathbf{J}_{0}}\right)=\sum_{\mathbf{I j}} u_{\mathbf{I} \mathbf{j}} \prod_{i: j^{i}=0} \nu^{i}\left(J_{0}^{i}\right) \otimes_{i: j^{i} \neq 0}\left(\Psi_{I^{i} j^{i}}\left(\chi_{J_{0}^{i}}\right)-\frac{\nu^{i}\left(J_{0}^{i}\right)}{\nu^{i}\left(I_{0}^{i}\right)} \Psi_{I^{i} j^{i}}\left(\chi_{I_{0}^{i}}\right)\right)
$$

Using the same arguments as in Lemma 3, we get that in the above series we will have nonzero terms only for those $\mathbf{I}, \mathbf{j}$ which for all $i=1, \ldots, n$ satisfy one of the conditions:

1) $j^{i} \neq 0, J_{0}^{i}<I^{i} \leq \sup \left(J_{0}^{i}, I_{0}^{i}\right)$;

2) $j^{i} \neq 0, I_{0}^{i}<I^{i} \leq \sup \left(J_{0}^{i}, I_{0}^{i}\right)$

3) $j^{i}=0, I^{i}=I_{0}^{i}$.

Since the number of $\mathbf{I}, \mathbf{j}$ satisfying the above conditions is finite, the series (6) converges in $D^{\prime}(X)$.

Applying ([6]) to $\overline{\Psi_{\mathbf{I j}}}$ we get (77).

An application of Lemma 7 proves that an arbitrary $u \in D^{\prime}(X)$ satisfying (17) has the form (6). This finishes the proof of the lemma.

\section{Multidimensional pseudodifferential operators}

In this section we introduce multidimensional ultrametric pseudodifferential operators. Let us consider the integral operators (linear combinations of one-dimensional operators) in $L^{2}(X, \nu)$ :

$$
T_{i} f=\int T_{i}\left(\sup \left(x^{i}, y^{i}\right)\right)\left(f\left(x^{1}, \ldots, x^{i}, \ldots, x^{n}\right)-f\left(x^{1}, \ldots, y^{i}, \ldots, x^{n}\right)\right) d \nu^{i}\left(y^{i}\right) .
$$

We call a multidimensional ultrametric pseudodifferential operator the following polynomial combination

$$
T=\sum_{k=1}^{m} \sum_{i_{1} \ldots i_{k}=1}^{n} a_{i_{1} \ldots i_{k}}^{(k)} T_{i_{1}} \ldots T_{i_{k}}
$$

where $a_{i_{1} \ldots i_{k}}^{(k)}$ are complex numbers.

In particular, we will consider operators of the form

$$
T^{(1)}=\sum_{i=1}^{n} a_{i} T_{i}, \quad T^{(2)}=\sum_{i j=1}^{n} a_{i j} T_{i} T_{j} .
$$

Since the operators $T_{i}$ are diagonal in the wavelet basis

$$
T_{i} \Psi_{\mathbf{I j}}=\lambda_{I^{i}} \Psi_{\mathbf{I} \mathbf{j}}
$$

the multidimensional pseudodifferential operators will be diagonal in the basis of wavelets and the corresponding eigenvalues for $\Psi_{\mathbf{I j}}$ depend only on $\mathbf{I}$ :

$$
T \Psi_{\mathbf{I j}}=\left[\sum_{k=1}^{m} \sum_{i_{1} \ldots i_{k}=1}^{n} a_{i_{1} \ldots i_{k}}^{(k)} \lambda_{I^{i_{1}}} \ldots \lambda_{I^{i_{k}}}\right] \Psi_{\mathbf{I j}}=\lambda_{\mathbf{I}} \Psi_{\mathbf{I} \mathbf{j}}
$$


Definition 9 We will say that a vertex $\mathbf{I} \in \mathcal{T}$ is characteristic for the multidimensional pseudodifferential operator $T$, if the eigenvalue $\lambda_{\mathbf{I}}$ of the operator $T$ is equal to zero.

This definition might look strange, but actually it is a direct analogue of the standard definition of a characteristic for real differential operators. The vertex $\mathbf{I}=I^{1} \times \ldots \times I^{n}$ is characteristic when the corresponding vector of eigenvalues $\Lambda=\left(\lambda_{I^{i}}\right), i=1, \ldots, n$ lies in the kernel of the multilinear form

$$
A(\Lambda)=\sum_{k=1}^{m} \sum_{i_{1} \ldots i_{k}=1}^{n} a_{i_{1} \ldots i_{k}}^{(k)} \lambda_{I^{i_{1}}} \ldots \lambda_{I^{i_{k}}}
$$

which defines the operator $T$.

This is the direct analogue of the real case, see Appendix 1 for discussion. Moreover, in the simplest case where the space $X$ is the product $Q_{p}^{n}$ of $p$-adic fields, the corresponding wavelet in the simplest case will have the form of product of a character of $Q_{p}^{n}$ and product of characteristic functions of balls. This can be compared with the formulae (15), (16) of the Appendix 1.

\section{Cauchy problem in the multidimensional case}

In the present section we investigate Cauchy problems for ultrametric pseudodifferential equations in many dimensions. We consider the following Cauchy problem: let us find $u$, satisfying

$$
T u=f,
$$

where $T$ is an ultrametric pseudodifferential operator with the eigenvalues $\lambda_{\mathbf{I}}$ in the wavelet basis, $f \in D_{0}^{\prime}(X)$ lies in Lizorkin space of generalized functions, $u \in D^{\prime}(X)$ is a generalized function, which satisfies the initial conditions (7) for some vertex $\mathbf{I}_{0} \in \mathcal{T}$ with the positive measure $\nu\left(\mathbf{I}_{0}\right)$.

We have the following necessary conditions for solvability of the above Cauchy problem: if $\lambda_{\mathbf{I}}=0$ for some $\mathbf{I} \in \mathcal{T}$ then $f_{\mathbf{I} \mathbf{j}}=f\left(\overline{\Psi_{\mathbf{I j}}}\right)=0$ for all corresponding ultrametric wavelets $\Psi_{\mathbf{I j}}$.

We have the following ultrametric analogue of the Kovalevskaya theorem.

Theorem 10 Let the multidimensional ultrametric pseudodifferential operator $T$ have the form

$$
T=\sum_{k=1}^{m} \sum_{i_{1} \ldots i_{k}=1}^{n} a_{i_{1} \ldots i_{k}}^{(k)} T_{i_{1}} \ldots T_{i_{k}},
$$

where $T_{i}$ are one-dimensional ultrametric pseudodifferential operators.

Consider the Cauchy problem

$$
T u=f
$$

where $f \in D_{0}^{\prime}(X)$, with $u \in D^{\prime}(X)$ satisfying the set of initial conditions

$$
u\left(\overline{\Psi_{\mathbf{I j}}}\right)=u_{\mathbf{I} \mathbf{j}} \prod_{i: j^{i}=0} \nu^{i}\left(I_{0}^{i}\right)
$$


for those $\mathbf{I}, \mathbf{j}$ for which at least one of the $j^{i}, i=1, \ldots, n$ will be equal to zero.

Assume that if $\mathbf{I}$ is characteristic, i.e. the corresponding eigenvalue $\lambda_{\mathbf{I}}$ of the operator $T$ in the basis of multidimensional ultrametric wavelets is equal to zero:

$$
\lambda_{\mathbf{I}}=\sum_{k=1}^{m} \sum_{i_{1} \ldots i_{k}=1}^{n} a_{i_{1} \ldots i_{k}}^{(k)} \lambda_{I^{i_{1}}} \ldots \lambda_{I^{i_{k}}}=0
$$

then $f_{\mathbf{I} \mathbf{j}}=f\left(\overline{\Psi_{\mathbf{I}}}\right)=0$ for all $\mathbf{j}$ (here $\Psi_{\mathbf{I} \mathbf{j}}$ are ultrametric wavelets, i.e. $j^{i}$ can not be equal to zero).

Then the solution $u$ of the Cauchy problem (10) does exist, is unique, belongs to $D^{\prime}(X)$ and is given by the series over ultrametric wavelets

$$
\begin{aligned}
& \sum_{\mathbf{I j}} u_{\mathbf{I j}} \otimes_{i: j^{i} \neq 0}\left(\Psi_{I^{i} j^{i}}-\frac{1}{\nu^{i}\left(I_{0}^{i}\right)} \Psi_{\mathbf{I j}}\left(\chi_{I_{0}^{i}}\right)\right)+ \\
+ & \sum_{\mathbf{I j}} \frac{1}{\lambda_{\mathbf{I}}} f_{\mathbf{I j}} \otimes_{i: j^{i} \neq 0}\left(\Psi_{I^{i} j^{i}}-\frac{1}{\nu^{i}\left(I_{0}^{i}\right)} \Psi_{\mathbf{I j}}\left(\chi_{I_{0}^{i}}\right)\right)+ \\
& +\sum_{\mathbf{I j}} u_{\mathbf{I j}} \otimes_{i: j^{i} \neq 0}\left(\Psi_{I^{i} j^{i}}-\frac{1}{\nu^{i}\left(I_{0}^{i}\right)} \Psi_{\mathbf{I j}}\left(\chi_{I_{0}^{i}}\right)\right) .
\end{aligned}
$$

Here summation in the first sum runs over the initial conditions (the corresponding $u_{\mathbf{I j}}$ are given by (11)); summation in the second sum runs over non-characteristic I; summation in the third sum runs over the characteristic $\mathbf{I}$ (the corresponding $u_{\mathbf{I j}}$ are arbitrary).

Proof By Lemma 8, any generalized function satisfying (11) has the form (6). Substituting this series into (10) we get for the case where $\mathbf{I}$ is not characteristic

$$
u_{\mathrm{Ij}}=\frac{1}{\lambda_{\mathbf{I}}} f_{\mathbf{I j}} \text {. }
$$

This implies for $u$ the expression

$$
\begin{aligned}
& u=\sum_{\mathbf{I j}} u_{\mathbf{I j}} \otimes_{i: j^{i} \neq 0}\left(\Psi_{I^{i} j^{i}}-\frac{1}{\nu^{i}\left(I_{0}^{i}\right)} \Psi_{\mathbf{I j}}\left(\chi_{I_{0}^{i}}\right)\right)+ \\
& +\sum_{\mathbf{I j}} \frac{1}{\lambda_{\mathbf{I}}} f_{\mathbf{I j}} \otimes_{i: j^{i} \neq 0}\left(\Psi_{I^{i} j^{i}}-\frac{1}{\nu^{i}\left(I_{0}^{i}\right)} \Psi_{\mathbf{I j}}\left(\chi_{I_{0}^{i}}\right)\right)+ \\
& +\sum_{\mathbf{I j}} u_{\mathbf{I j}} \otimes_{i: j^{i} \neq 0}\left(\Psi_{I^{i} j^{i}}-\frac{1}{\nu^{i}\left(I_{0}^{i}\right)} \Psi_{\mathbf{I j}}\left(\chi_{I_{0}^{i}}\right)\right)
\end{aligned}
$$

Here the summation in the first sum runs over the initial conditions, i.e. over those $\mathbf{I}, \mathbf{j}$ for which at least one $j^{i}, i=1, \ldots, n$ is equal to zero. The corresponding $u_{\mathbf{I j}}$ are given by the initial conditions (11).

The summation in the second sum runs over the non-characteristic vertices $\mathbf{I}$. The summation in the third sum runs over characteristic vertices $\mathbf{I}$, and the corresponding $u_{\mathbf{I j}}$ are arbitrary. 
This finishes the proof of the theorem.

We see that the possible source of problems for existence and uniqueness of the solution for Cauchy problem is the case where some vertex $\mathbf{I}$ is characteristic for the operator $T$.

Example We shall discuss the case of the ultrametric analogue of the propagating wave solution for the wave equation. Consider the two dimensional case where $X=X^{1} \times X^{2}$, $\nu=\nu^{1} \times \nu^{2}$, and the pseudodifferential operator has the form

$$
T=T_{1}-T_{2}
$$

which implies $\lambda_{\mathbf{I}}=\lambda_{I^{1}}-\lambda_{I^{2}}$.

Consider the Cauchy problem

$$
T u=0, \quad u\left(\chi_{\mathbf{I}_{0}}\right)=0 .
$$

Assume that the vertex $\mathbf{I}$ is characteristic (i.e. $\lambda_{I^{1}}=\lambda_{I^{2}}$ ) and $\mathbf{I}_{0} \cap \mathbf{I}=\emptyset$. Then any $u \in V^{0}(\mathbf{I})$ will be a solution of the Cauchy problem.

In the case under consideration the solution of the Cauchy problem does exist but it is not unique. We can consider this solution as an ultrametric analogue of the propagating wave solution for the wave equation. Note that this propagating wave propagates inside $\mathbf{I}=I^{1} \times I^{2}$ and therefore is localized both in space and in scale.

\section{Appendix 1: Characteristics}

We recall here the standard definitions. Consider the linear partial differential equation of the order $m$ :

$$
\sum_{|\alpha| \leq m} a_{\alpha}(x) \partial_{x}^{\alpha} u(x)=f(x)
$$

Here $\alpha=\left(\alpha_{1}, \ldots, \alpha_{n}\right)$ is a multiindex, $|\alpha|=\sum_{i=1}^{n} \alpha_{i}$ is the order of the multiindex,

$$
\partial_{x}^{\alpha}=\prod_{i=1}^{n} \frac{\partial^{\alpha_{i}}}{\partial x_{i}^{\alpha_{i}}} .
$$

We say that non-zero vector $\xi=\left(\xi_{1}, \ldots, \xi_{n}\right)$ has a characteristic direction in the point $x$, if the corresponding characteristic polynomial of equation (14) is equal to zero

$$
\sum_{|\alpha|=m} a_{\alpha}(x) \xi^{\alpha}=0, \quad \xi^{\alpha}=\prod_{i=1}^{n} \xi_{i}^{\alpha_{i}}
$$

A hypersurface is said to be characteristic, if the normal vector to this surface at any point has a characteristic direction.

Let us note that application of the terms of higher order of the differential operator at the LHS of the equation (14) to the function

$$
\phi(x)=e^{i \sum_{l=1}^{n} \xi_{l} x_{l}}
$$


gives

$$
D \phi(x)=i^{|\alpha|} \phi(x) \sum_{|\alpha|=m} a_{\alpha}(x) \xi^{\alpha}, \quad D=\sum_{|\alpha| \leq m} a_{\alpha}(x) \partial_{x}^{\alpha},
$$

which is proportional to the characteristic polynomial.

Note that the function $\phi$ is a character of the linear space $R^{n}$ and is an eigenfunction (in the sense of generalized functions) of the differentiation operators $\partial_{x_{i}}$, and the characteristic polynomial in (16) is a polynomial over the corresponding eigenvalues.

\section{Appendix 2: Ultrametric analysis}

In this Section we summarize the results on ultrametric analysis, which may mainly be found in [10], [11], [12]. We discuss ultrametric wavelet analysis, analysis of ultrametric pseudodifferential operators (PDO), and ultrametric distribution theory.

\subsection{Ultrametric pseudodifferential operators}

Definition 11 An ultrametric space is a metric space with the ultrametric $d(x, y)$ (where $d(x, y)$ is called the distance between $x$ and $y)$, i.e. a function of two variables, satisfying the properties of positivity and non degeneracy

$$
d(x, y) \geq 0, \quad d(x, y)=0 \quad \Longrightarrow \quad x=y
$$

symmetricity

$$
d(x, y)=d(y, x)
$$

and the strong triangle inequality

$$
d(x, y) \leq \max (d(x, z), d(y, z)), \quad \forall x, y, z .
$$

We say that an ultrametric space $X$ is regular, if this space satisfies the following properties:

1) The set of all the balls of nonzero diameter in $X$ is finite or countable;

2) For any decreasing sequence of balls $\left\{D^{(k)}\right\}, D^{(k)} \supset D^{(k+1)}$, the diameters of the balls tend to zero;

3) Any ball of non-zero diameter is a finite union of maximal subballs.

Ultrametric spaces are dual to directed trees. Below we describe some part of the duality construction.

For a regular ultrametric space $X$ consider the set $\mathcal{T}(X)$, which contains all the balls in $X$ of nonzero diameters, and the balls of zero diameter which are maximal subbals in balls of nonzero diameters. This set possesses a natural structure of a directed tree. Two vertices $I$ and $J$ in $\mathcal{T}(X)$ are connected by an edge if the corresponding balls are ordered by inclusion, say $I \supset J$ (i.e. one of the balls contain the other), and there are no intermediate balls between $I$ and $J$. 
The partial order in $\mathcal{T}(X)$ is defined by inclusion of balls, this partial order is a direction. We recall that a partially ordered set is a directed set (and a partial order is a direction), if for any pair of elements there exists the unique supremum with respect to the partial order.

A vertex $I$ of a directed tree has by definition a branching index $p_{I}$, if the corresponding ball contains $p_{I}$ maximal subballs.

The supremum

$$
\sup (x, y)=I
$$

of the points $x, y \in X$ is the minimal ball $I$ in $X$, containing both points.

Consider a $\sigma$-additive Borel measure $\nu$ with countable or finite basis on a regular ultrametric space $X$. We study the ultrametric pseudodifferential operator (shortly written PDO) of the form considered in [10], [11], [12], i.e.:

$$
T f(x)=\int T(\sup (x, y))(f(x)-f(y)) d \nu(y)
$$

Here $T(I)$ is some complex valued function on the tree $\mathcal{T}(X)$.

\subsection{Ultrametric wavelets}

Build a basis in the space $L^{2}(X, \nu)$ of complex valued functions on a regular ultrametric space $X$ which are quadratically integrable with respect to the measure $\nu$. We will call this basis the basis of ultrametric wavelets.

Denote by $V(I)$ the space of functions on $X$, generated by characteristic functions of the maximal subballs in the ball $I$ of nonzero diameter. Correspondingly, $V^{0}(I)$ is the subspace of codimension 1 in $V(I)$ of functions with zero mean with respect to the measure $\nu$. The spaces $V^{0}(I)$ for different $I$ are orthogonal. The dimension of the space $V^{0}(I)$ is equal to $p_{I}-1$ (if $\nu\left(I_{j}\right) \neq 0$ for all maximal subballs $I_{j}$ in $I$ ).

We introduce in the space $V^{0}(I)$ some orthonormal basis $\left\{\psi_{I j}\right\}$. If the measures of all maximal subballs in $I$ are positive, the index $j$ can take values $1, \ldots, p_{I}-1$. The next theorem shows how to construct the orthonormal basis in $L^{2}(X, \nu)$, taking the union of bases $\left\{\psi_{I j}\right\}$ in the spaces $V^{0}(I)$ over all non minimal $I$ (equivalently, over all balls $I$ of non-zero diameters).

Theorem 12 1) Let the measure $\nu(X)$ of the regular ultrametric space $X$ is infinite. Then the set of functions $\left\{\psi_{I j}\right\}$, where $I$ runs over all non minimal vertices of the tree $\mathcal{T}(X)$ is an orthonormal basis in $L^{2}(X, \nu)$.

2) Let the measure $\nu(X)$ of the regular ultrametric space $X$ is finite and is equal to $\nu(X)=A$. Then the set of functions $\left\{\psi_{I j}, A^{-\frac{1}{2}}\right\}$, where $I$ runs over all non minimal vertices of the tree $\mathcal{T}(X)$ is an orthonormal basis in $L^{2}(X, \nu)$.

The basis introduced in the present theorem will be called the basis of ultrametric wavelets.

The next theorem shows that the basis of ultrametric wavelets is the basis of eigenvectors for ultrametric pseudodifferential operators. 
Theorem 13 Let the following series converge absolutely:

$$
\sum_{J>R} T(J)(\nu(J)-\nu(J(R)))<\infty
$$

for some ball $R$.

Then the ultrametric pseudodifferential operator

$$
T f(x)=\int T(\sup (x, y))(f(x)-f(y)) d \nu(y)
$$

has a dense domain in $L^{2}(X, \nu)$ and ultrametric wavelets from Theorem[12 are eigenfunctions of $T$ :

$$
T \psi_{I j}(x)=\lambda_{I} \psi_{I j}(x)
$$

with the eigenvalues:

$$
\lambda_{I}=T(I) \nu(I)+\sum_{J>I} T(J)(\nu(J)-\nu(J(I)))
$$

Here $J(I)$ is the maximal subball in $J$ which contains $I$.

Also the operator $T$ maps constants into zero.

\subsection{Distributions}

Here we discuss the spaces of (complex valued) test and generalized functions (or distributions) on a regular ultrametric space $X$. This construction is an analogue of the construction of the Bruhat-Schwartz space in the $p$-adic case, which can be found in [1].

Definition 14 A function $f$ on an (ultrametric) space $X$ is called locally constant, if for any arbitrary point $x \in X$ there exists a positive number $r$ (depending on $x$ ), such that the function $f$ is constant on the ball with the center in $x$ and the radius $r$ :

$$
f(x)=f(y), \quad \forall y: d(x, y) \leq r .
$$

In particular, the characteristic function $\chi_{I}$ of a ball $I$ is locally constant. Any locally constant function is continuous. The next definition is an analogue of a known definition for the Bruhat-Schwartz space in the $p$-adic case.

Definition 15 The space of test functions $D(X)$ on an ultrametric space $X$ is defined as the space of locally constant functions with compact support.

We remind that a filtration of a set $A$ by a partially ordered set $B$ is a map $\phi$ which maps $B$ to the partially ordered (by inclusion) set of subsets in $A$, and $\phi$ satisfies the following conditions:

1) the map $\phi$ conserves the partial order, i.e. if $b<b^{\prime}, b, b^{\prime} \in B$, then there exists an embedding of $\phi(b)$ into $\phi\left(b^{\prime}\right)$;

2 ) any element of the set $A$ lies in the image of some element of $B$.

In the case of filtrated linear spaces we will consider filtrations by families of linear subspaces, i.e. an embedding of $\phi(b)$ into $\phi\left(b^{\prime}\right)$ for $b<b^{\prime}, b, b^{\prime} \in B$ should be linear.

Consider the tree $\mathcal{T}=\mathcal{T}(X)$ of balls in an ultrametric space $X$. Introduce the filtration of the space $D(X)$ of test functions by finite dimensional linear subspaces $D(\mathcal{S})$, where $\mathcal{S} \subset \mathcal{T}$ is a finite directed subtree of the following "regular" form: 
Definition 16 A finite subtree $\mathcal{S}$ in a directed tree $\mathcal{T}(X)$ is called of the regular type, iff:

1) If $\mathcal{S}$ contains the balls $I$ and $J$, then it contains $\sup (I, J)$;

2) If $\mathcal{S}$ contains the balls $I$ and $J: I \subset J$, then $\mathcal{S}$ contains all the balls $L: I \subset L \subset J$;

3) If $\mathcal{S}$ contains the balls $I$ and $J$, where $J$ is a maximal subball in $I$, then it contains all the maximal subballs in $I$.

We denote by $\chi_{J}$ a characteristic function of a ball $J$.

Definition 17 For a subtree $\mathcal{S} \subset \mathcal{T}$ of the regular type consider the space $D(\mathcal{S})$, which is the linear span of characteristic functions $\chi_{J}$ with $J \in \mathcal{S}$.

The space $D(\mathcal{S})$ has the natural topology (which can be described as the topology of pointwise convergence), since $D(\mathcal{S})$ is finite dimensional. The space $D(X)$ of test functions on $X$ is the inductive limit of spaces $D(\mathcal{S})$ :

$$
D(X)=\lim _{\operatorname{ind}} \rightarrow \mathcal{T} D(\mathcal{S})
$$

Since all spaces $D(\mathcal{S})$ are finite dimensional, the restriction of the topology of $D(\mathcal{S})$ to any subspace $D\left(\mathcal{S}_{0}\right), \mathcal{S} \supset \mathcal{S}_{0}$, coincides with the original topology of $D\left(\mathcal{S}_{0}\right)$. Thus the inductive limit (20) is, in fact, the strict inductive limit [17] p.57. By proposition 6.5, [17], p.59, a set $B$ in a strict inductive limit of a countable family of locally convex spaces $\left\{E_{n}\right\}$ is bounded iff there exists $n$ such that $B \subset E_{n}$ and bounded in it. This implies the proposition below.

Proposition 18 The sequence $\left\{f_{n}\right\} \in D(X)$ converges, if this sequence lies in some subspace $D(\mathcal{S})$ of the filtration and converges (and the convergence in the finite dimensional space $D(\mathcal{S})$ is defined uniquely).

By corollary of theorem 7.4, [17], p.103, we have the following proposition.

Proposition 19 The space $D(X)$ is nuclear.

Definition 20 A distribution (or a generalized function) on $X$ is a linear functional on the space $D(X)$ of test functions.

It is easy to see that this functional automatically will be continuous (since convergence in the space of test functions is defined through the convergence in finite dimensional subspaces). The linear space of generalized functions will be denoted by $D^{\prime}(X)$. The convergence in $D^{\prime}(X)$ is defined as a weak convergence of functionals. Thus $D^{\prime}(X)$ is conjugated to $D(X)$ with the weak topology.

Acknowledgments One of the authors (S.K.) would like to thank I.V.Volovich, V.S.Vladimirov, A.Yu.Khrennikov and V.M.Shelkovich for fruitful discussions and valuable comments. He gratefully acknowledges being partially supported by the grant DFG Project 436 RUS 113/809/0-1, by the grants of The Russian Foundation for Basic Research RFFI 05-0104002-NNIO-a and RFFI 05-01-00884-a, by the grant of the President of Russian Federation for the support of scientific schools NSh 6705.2006.1 and by the Program of the Department of Mathematics of Russian Academy of Science "Modern problems of theoretical mathematics".

S.K. is grateful to IZKS (The Interdisciplinary Center for Complex Systems) of the University of Bonn for kind hospitality. 


\section{References}

[1] V.S. Vladimirov, I.V. Volovich, Ye.I. Zelenov p-Adic analysis and mathematical physics, World Scientific, Singapore, 1994 (See also Nauka, Moscow, 1994, in Russian).

[2] A.Yu.Khrennikov, M.Nilsson P-adic Deterministic and Random Dynamics, Kluwer Academic, Dordrecht-Boston-London, 2004.

[3] A.N.Kochubei Pseudo-Differential Equations and Stochastics over Non-Archimedean Fields. Marcel Dekker, New York, 2001.

[4] S.Albeverio, W.Karwowski A random walk on $p$-adics - the generator and its spectrum // Stoch. Processes Appl. 1994. V.53. no.1. P.1-22.

[5] S.Albeverio, X.Zhao On the relation between different constructions of random walks on p-adics // Markov Processes and Related fields. 2000. V.6. P.239-255.

[6] K.Yasuda Additive processes on local fields // J.Math.Sci.Univ.Tokyo. 1996. V.3. P.629654 .

[7] S.Evans Local fields, Gaussian measures, and Brownian motions // Topics in probability and Lie groups: boundary theory, CRM Proc. Lecture Notes. 2001. V.28. P.11-50. Amer. Math. Soc., Providence, RI.

[8] S.V.Kozyrev Wavelet analysis as a $p$-adic spectral analysis // Russian Math. Izv. 2002. V. 66. No 2. P. 367-376. http://arxiv.org/abs/math-ph/0012019

[9] J.J. Benedetto, R.L. Benedetto A wavelet theory for local fields and related groups // The Journal of Geometric Analysis. 2004. V.14. N.3. P.423-456.

[10] A.Yu.Khrennikov, S.V.Kozyrev Pseudodifferential operators on ultrametric spaces and ultrametric wavelets // Izvestiya: Mathematics. 2005. V.69. N.5. P.989-1003.

[11] A.Yu.Khrennikov, S.V.Kozyrev Wavelets on ultrametric spaces// Applied and Computational Harmonic Analysis. 2005. V.19. P.61-76.

[12] S.V.Kozyrev Wavelets and spectral analysis of ultrametric pseudodifferenial operators // Sbornik Mathematics. 2007. V.198. N.1.P.103-126.

[13] V.M.Shelkovich, M.Skopina p-Adic Haar multiresolution analysis // http://arxiv.org/abs/0704.0736

[14] S.Albeverio, S.V.Kozyrev Coincidence of the continuous and discrete $p$-adic wavelet transforms // http://arxiv.org/abs/math-ph/0702010

[15] S.V.Kozyrev $p$-Adic pseudodifferenial operators and $p$-adic wavelets // Theoretical and Mathematical Physics. 2004. V.138. N.3. P.322-332. 
[16] S.Albeverio, A.Yu.Khrennikov, V.M.Shelkovich Harmonic analysis in the $p$-adic Lizorkin spaces: fractional operators, pseudo-differential equations, $p$-adic wavelets, Tauberian theorems // The Journal of Fourier Analysis and Applications. 2006. V.12. N.4. P.393-425.

[17] H.H.Schaefer Topological Vector Spaces. New York: Springer Verlag, 1999. 